\title{
Tolosa-Hunt Syndrome, the Hunt for One in a Million
}

\author{
Zufe Rizvi $^{1}$, Asia Filatov ${ }^{1}$, Marc Swerdloff ${ }^{2}$ \\ 1. Neurology, Charles E. Schmidt College of Medicine, Florida Atlantic University, Boca Raton, USA 2. Neurology, \\ Marcus Neuroscience Institute, Boca Raton, USA
}

Corresponding author: Zufe Rizvi, zuferizvi@gmail.com

\begin{abstract}
Tolosa-Hunt Syndrome (THS) is a rare disorder characterized by severe unilateral periorbital headache, painful eye movements, and double vision. This syndrome affects males and females equally in their forties to fifties. THS is caused by granulomatous inflammation of the cavernous sinus, usually involving CN III, IV, and VI. We herein report a case of Tolosa-Hunt Syndrome in an 18-year-old female who was initially misdiagnosed as idiopathic intracranial hypertension. Initial treatment was ineffective and the delay may have allowed spread of the disease to involve CN V and VII. This case highlights the pitfalls which can lead to a delay in diagnosis and treatment.
\end{abstract}

Categories: Neurology, Ophthalmology, Radiology

Keywords: tolosa-hunt syndrome, cranial nerves, headache

\section{Introduction}

Tolosa-Hunt syndrome is a rare disorder with an estimated annual case of one individual per million per year [1]. The average age of onset is 40 years and above, with a minority of cases reported in children and young adults. We present a case of Tolosa-Hunt syndrome in an 18-year-old woman. The syndrome is characterized by a severe unilateral periorbital headache, pain with eye movement, double vision and sensory loss in the V1 branch of the trigeminal nerve which traverses the cavernous sinus where there is granulomatous inflammation [2]. While the syndrome is usually a temporally limited condition, relapses may occur requiring extended periods of immunosuppressive therapy.

Received 04/20/2020 Review began 05/10/2020 Review ended 05/15/2020 Published 05/22/2020

\section{() Copyright 2020}

Rizvi et al. This is an open access article distributed under the terms of the Creative Commons Attribution License CC-BY 4.0., which permits unrestricted use, distribution, and reproduction in any medium, provided the original author and source are credited.

\section{Case Presentation}

An 18-year-old female with a history of morbid obesity presented for evaluation of an intractable left-sided headache associated with left facial numbness, left eyelid ptosis and diplopia. Her headache was present for five weeks. Prior to this, she never had headaches. Three weeks prior to admission, she had a negative noncontrast CT head scan and a negative contrast enhanced MRI brain. She was noted to have an increased intracranial pressure with lumbar puncture revealing $35 \mathrm{~cm}$ cerebral spinal fluid (CSF) opening pressure. She was diagnosed with idiopathic intracranial hypertension (IIH). The patient was treated with acetazolamide, an anti-emetic, hydrochlorothiazide and a tapering dose of prednisone. She denied the use of birth control pills, retinoid acid or acne medications. Her headaches continued and a second diagnosis of complicated migraines was made. The patient was then prescribed topiramate and non-steroidal analgesics. Her headaches continued despite these two disparate treatments.

On presentation to our facility, she was suffering from a five-week long unremitting left periorbital stabbing headache. She recalled it was unrelieved by her initial spinal tap. Her physical exam showed left lid ptosis and left eye with a fifty percent adduction insufficiency (left partial third cranial-nerve paresis) and a fifty percent abduction insufficiency (left sixth cranial nerve paresis) and decreased pin sensation in the left V1 distribution. There was no meningismus. There was no papilledema OU (of both eyes) and spontaneous venular pulsations were noted on bedside fundoscopy consistent with normal intracranial pressure. Magnetic resonance angiography (MRA) of the head revealed high-grade stenosis of the left internal carotid artery through the cavernous sinus (Figure 1). Review of her initial MRI brain revealed an enlarged enhancing left cavernous sinus (Figures 2, 3). A repeat lumbar puncture revealed an opening pressure of 20 cm CSF with 66 lymphocytes consistent with an inflammatory disorder (see Table 1). Her history, physical exam, CSF findings and neuroimaging led to the diagnosis of Tolosa-Hunt syndrome. Neurosurgical consultation evaluating the internal carotid artery (ICA) stenosis did not consider her a candidate for endovascular intervention. Infectious Disease consultation advised that steroids were not contraindicated in the setting of CSF pleocytosis. She was placed on prednisone $80 \mathrm{mg}$ per os daily. Further workup included a negative magnetic resonance venography (MRV) and MRA neck. The patient dramatically improved after initiation of steroid therapy. She was discharged home on a steroid taper. Thirteen days later, before she could see a neuro-ophthalmologist, she presented with a left-sided facial palsy. Non-contrast CT head and contrast MRI brain were unchanged. Acyclovir $400 \mathrm{mg}$ twice per day (BID) for five days was added to her prednisone, now at $60 \mathrm{mg}$ per day. 


\section{Cureus}

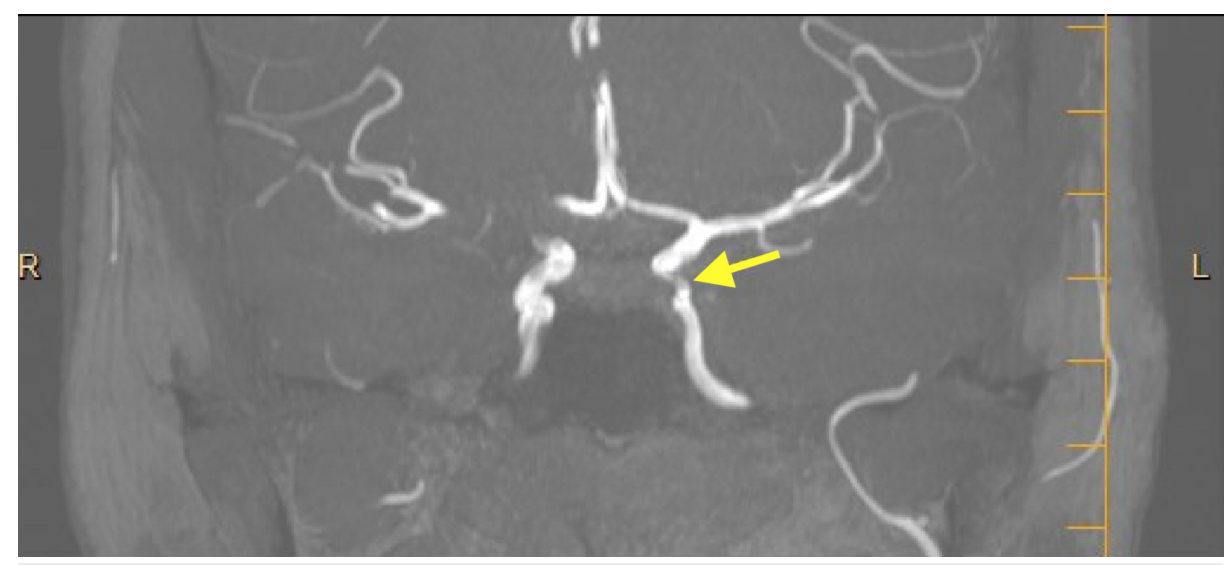

FIGURE 1: MRA of the head. Arrow indicating the area of high-grade stenosis of the Left Internal Carotid Artery through the cavernous sinus.

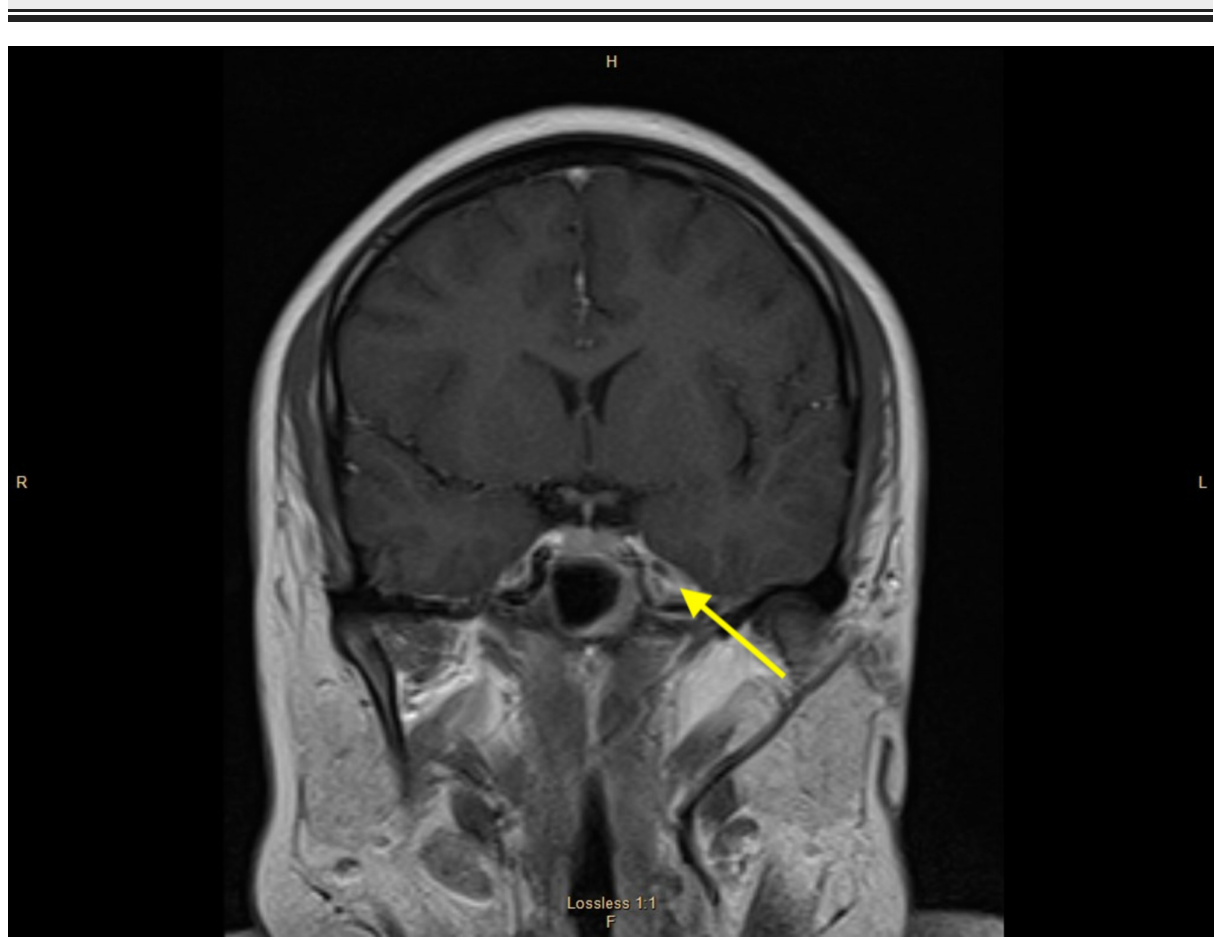

FIGURE 2: MRI brain. Here, the arrow points to an enlarged enhancing left cavernous sinus. 


\section{Cureus}

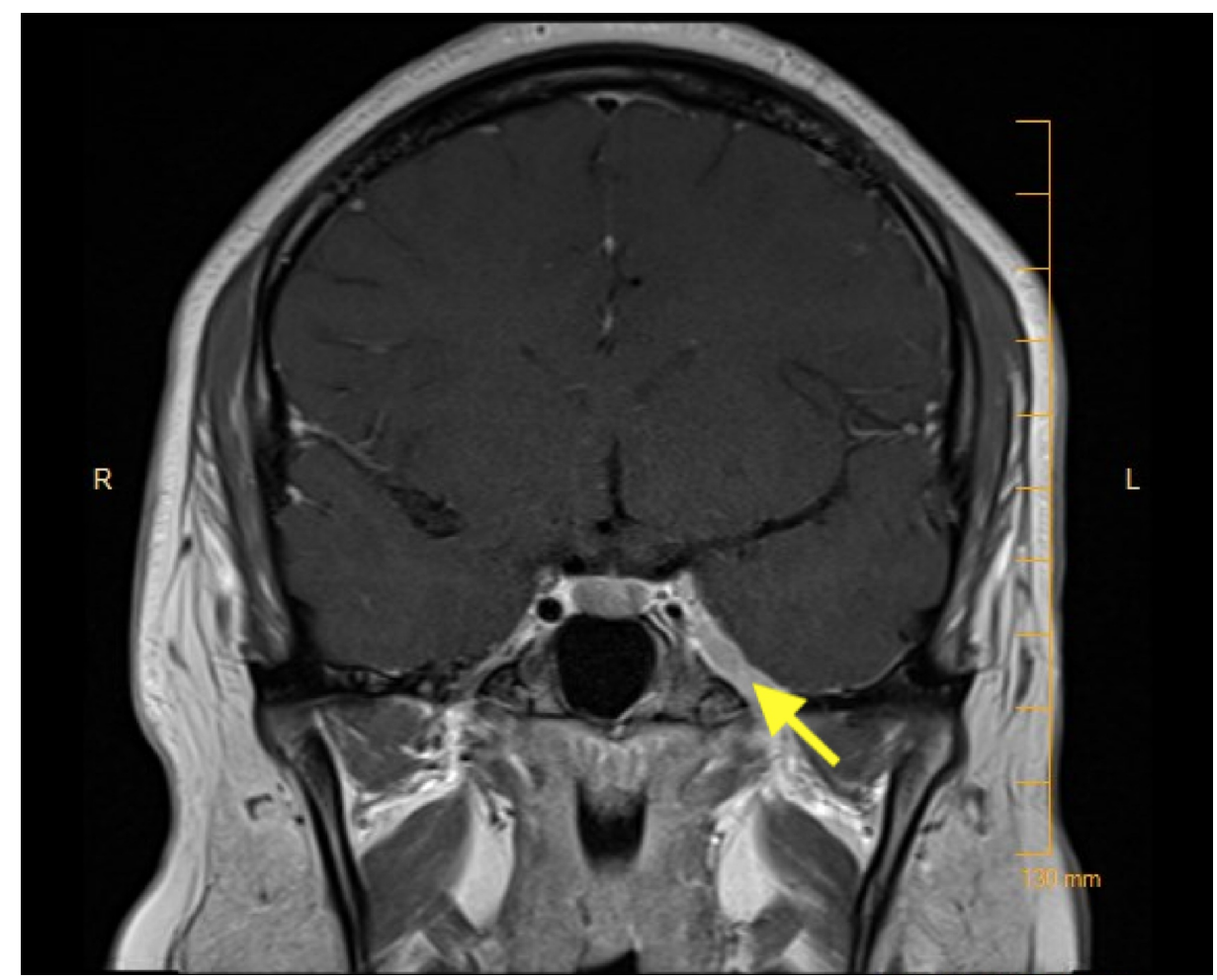

FIGURE 3: MRI brain. Another image indicating the enlarged and enhancing left cavernous sinus.

CSF Studies

Appearance

clear

Color

none

WBCs

66

RBCs

0

CSF Glucose

66

CSF Protein

44

Albumin

23

TABLE 1: Cerebral spinal fluid (CSF) studies.

\section{Discussion}

Tolosa-Hunt syndrome (THS) is a rare disorder characterized by unilateral ophthalmoplegia and severe unilateral headache. It is a neuro-immunologic disorder considered to be a diagnosis of exclusion. It has an incidence of one per million cases per year. THS affects men and women equally and is found in all decades. The average age of onset for Tolosa-Hunt syndrome is in the fifth decade of life. Affected patients often present with sudden onset of multiple cranial nerve palsies with diplopia, lid ptosis, facial numbness, and unilateral headache. It is an idiopathic granulomatous inflammation involving the cavernous sinus affecting the structures found within the sinus such as CN III, IV and VI. In some cases, involvement of CN V and VII has been reported. Diagnosis is made by noting the characteristic symptoms, clinical signs, and radiographic findings. Diagnostic criteria by the International Headache Society include unilateral headache plus MRI exam or biopsy showing granulomatous inflammation of either the cavernous sinus or superior orbital fissure, plus impairment of one or more of the oculomotor nerves. The criteria also require the headache to be on the same side as the involved sinus or preceded by oculomotor paresis. CSF analysis in Tolosa-Hunt 
syndrome is usually unremarkable but some cases display CSF pleocytosis. In those cases, meningitis must be considered.

Tolosa-Hunt syndrome has unique features that are important for medical practitioners to remember. Persistent periorbital pain may manifest itself before or after other signs [3]. The symptoms might also involve periarterial sympathetic nerves. If left untreated, the process may spread to other cranial nerves. Headache may present as severe intermittent frontal pain. If the optic nerve is involved, impaired visual acuity may be irreversible [4]. THS, which is rare, may also present in a myriad of ways making it a diagnostic challenge.

The differential diagnosis includes herpes zoster infection, bacterial sinusitis, or fungal infection [5]. Initially, our patient was diagnosed with idiopathic intracranial hypertension (IIH). Noticing the focal nature of her pain, absence of papilledema and the involvement of cranial nerves uncharacteristic of IIH (CN III and V) raised red flags. Elevation of the CSF opening pressure from a tense patient misdirected the diagnosis. Subtle expansion of the cavernous sinus was missed on initial radiological review. This case illustrates the difficulty in diagnosing a rare disease in a patient population which is rarely affected. Our patient's dramatic pain relief after steroid therapy was consistent with the diagnosis of THS [5]. In nearly half of patients however, long-term steroid treatment may be required to relieve recurrent symptoms.

\section{Conclusions}

This case illustrates the pitfalls which may occur in the diagnosis of Tolosa-Hunt syndrome presenting in an age group rarely affected. Tolosa-Hunt syndrome is a rare idiopathic granulomatous inflammation involving the cavernous sinus and the structures traversing it, including the internal carotid artery and cranial nerves. With treatment delay, it may spread to involve structures not within the cavernous sinus.

\section{Additional Information \\ Disclosures}

Human subjects: Consent was obtained by all participants in this study. Conflicts of interest: In compliance with the ICMJE uniform disclosure form, all authors declare the following: Payment/services info: All authors have declared that no financial support was received from any organization for the submitted work. Financial relationships: All authors have declared that they have no financial relationships at present or within the previous three years with any organizations that might have an interest in the submitted work. Other relationships: All authors have declared that there are no other relationships or activities that could appear to have influenced the submitted work.

\section{References}

1. Amrutkar C, Burton EV: Tolosa-Hunt Syndrome. StatPearls Publishing, Treasure Island; 2020.

2. Arshad A, Nabi S, Panhwar MS, Rahil A: Tolosa-Hunt syndrome: an arcane pathology of cavernous venous sinus. BMJ Case Rep. 2015, 2015:bcr2015210646. 10.1136/bcr-2015-210646

3. Hao R, He Y, Zhang H, Zhang W, Li X, Ke Y: The evaluation of ICHD-3 beta diagnostic criteria for TolosaHunt syndrome: a study of 22 cases of Tolosa-Hunt syndrome. Neurol Sci. 2015, 36:899-905. 10.1007/s10072-015-2124-2

4. Höhne C, Schuh E, Kümpfel T, Straube A: Cryopyrin-associated periodic fever syndrome manifesting as Tolosa-Hunt syndrome. Cephalalgia. 2016, 36:1392-1396. 10.1177/0333102416629239

5. Takasuna H, Sasaki R, Shiraishi M, et al.: Steroid-resistant Tolosa-Hunt syndrome with a de novo intracavernous aneurysm: a case report. Surg Neurol Int. 2016, 7:S779-S784. 10.4103/2152-7806.193925 